\title{
ON SUMS OF RUDIN-SHAPIRO COEFFICIENTS II
}

\author{
John Brillhart, Paul Erdös and Patrick Morton
}

Let $\{a(n)\}$ be the Rudin-Shapiro sequence, and let $s(n)=$ $\sum_{k=0}^{n} a(k)$ and $t(n)=\sum_{k=0}^{n}(-1)^{k} a(k)$. In this paper we show that the sequences $\{s(n) / \sqrt{n}\}$ and $\{t(n) / \sqrt{n}\}$ do not have cumulative distribution functions, but do have logarithmic distribution functions (given by a specific Lebesgue integral) at each point of the respective intervals $[\sqrt{3 / 5}$, $\sqrt{6}]$ and $[0, \sqrt{3}]$. The functions $a(x)$ and $s(x)$ are also defined for real $x \geq 0$, and the function $[s(x)-a(x)] / \sqrt{x}$ is shown to have a Fourier expansion whose coefficients are related to the poles of the Dirichlet series $\sum_{n=1}^{\infty} a(n) / n^{\tau}$, where $\operatorname{Re} \tau>\frac{1}{2}$.

1. Introduction. In this paper we are concerned with the RudinShapiro sums

$$
\begin{gathered}
s(x)=\sum_{k=0}^{[x]} a(k), \\
t(x)=\sum_{k=0}^{[x]}(-1)^{k} a(k),
\end{gathered}
$$

where the numbers $a(k)$ are defined recursively by

$$
a(2 k)=a(k), \quad a(2 k+1)=(-1)^{k} a(k), \quad k \geq 0, a(0)=1 .
$$

An explicit formula for $a(k)$ is given by

$$
a(k)=(-1)^{e(k)},
$$

where $e(k)=\sum_{i=0}^{s-1} \varepsilon_{i} \varepsilon_{i+1}$ and $k=\sum_{i=0}^{s} \varepsilon_{i} 2^{i}, \varepsilon_{i}=0$ or 1. (See [1], Satz 1.)

The properties of these sums have been developed in [1], where it is shown that

$$
\begin{gathered}
\sqrt{\frac{3}{5}}<\frac{s(n)}{\sqrt{n}}<\sqrt{6}, \\
0 \leq \frac{t(n)}{\sqrt{n}}<\sqrt{3},
\end{gathered}
$$

for $n \geq 1$, and that the sequences $\{s(n) / \sqrt{n}\}$ and $\{t(n) / \sqrt{n}\}$ are dense in the intervals $[\sqrt{3 / 5}, \sqrt{6}]$ and $[0, \sqrt{3}]$. 
Here we study the quotients $s(n) / \sqrt{n}$ and $t(n) / \sqrt{n}$ further by introducing the limit functions

$$
\begin{aligned}
& \lambda(x)=\lim _{k \rightarrow \infty} \frac{s\left(4^{k} x\right)}{\sqrt{4^{k} x}}, \\
& \mu(x)=\lim _{k \rightarrow \infty} \frac{t\left(4^{k} x\right)}{\sqrt{4^{k} x}},
\end{aligned}
$$

which are defined for $x>0$. We show that $\lambda(x)$ and $\mu(x)$ are continuous functions of $x$, but are non-differentiable almost everywhere. Since $\lambda$ and $\mu$ satisfy the functional equations

$$
\lambda(4 x)=\lambda(x), \quad \mu(4 x)=\mu(x),
$$

the curves $\{(x, \lambda(x)) ; 1 \leq x \leq 4\}$ and $\{(x, \mu(x)) ; 1 \leq x \leq 4\}$ represent the limiting behavior of the quotients $s(n) / \sqrt{n}$ and $t(n) / \sqrt{n}$ on the intervals $\left[4^{k}, 4^{k+1}-1\right]$, as $k \rightarrow \infty$. (See Figure 1 in $\S 4$.)

Equation (1.7) implies also that $\lambda(x)$ has a Fourier series expansion of the form

$$
\lambda(x)=\sum_{n=-\infty}^{\infty} c_{n} e^{\pi i n \log x / \log 2},
$$

where $c_{n} \in \mathbf{C}$. This series is $(C, 1)$ summable to $\lambda(x)$ for all $x>0$, and is convergent in the usual sense for almost all $x>0$. In fact, we are able to give an explicit set on which (1.8) is convergent, the set of $x>0$ which are simply normal to the base 4 . (See $\S 4,5$, and [6].) This allows us to say, for example, that (1.8) converges when $x=m+\frac{9}{85}$, where $m$ is a non-negative integer.

Formula (1.8) then leads to an explicit formula for $s(x)$ of the form

$$
s(x)=\sqrt{x} \sum_{n=-\infty}^{\infty} c_{n} x^{\pi i n / \log 2}+a(x), \quad x>0,
$$

where $a(x)$ is an extension of the function $a(n)$, defined for real arguments $x \geq 0$. The function $a(x)$ is bounded, and has an explicit representation in terms of the digits of $x$ to the base 4. Formula (1.9) accounts for the roughly "periodic" behavior of the sequence $\{s(n) / \sqrt{n}\}$.

We show further that the Fourier coefficients $c_{n}$ are related to the poles of the function $\eta(\tau)$ defined by the Dirichlet series

$$
\eta(\tau)=\sum_{n=1}^{\infty} \frac{a(n)}{n^{\tau}}, \quad \operatorname{Re} \tau>\frac{1}{2} .
$$


This function has a meromorphic continuation to the whole complex plane, and its only poles in the half-plane $\operatorname{Re} \tau>0$ occur among the points $\gamma_{n}=1 / 2+\pi n i / \log 2, n \in \mathbf{Z}$. We prove that $\gamma_{n} c_{n}$ is equal to the residue of $\eta(\tau)$ at $\tau=\gamma_{n}$, and use this fact to show that infinitely many of the points $\gamma_{n}$ are poles of $\eta(\tau)$. This is seen to be a consequence of the fact that $\lambda(x)$ is not everywhere differentiable.

Finally, we use $\lambda(x)$ to prove the non-existence of the cumulative (or natural) distribution function of the sequence $\{s(n) / \sqrt{n}\}$ on the interval $(\sqrt{3 / 5}, \sqrt{6})$. By this we mean the $\operatorname{limit}_{\lim _{x \rightarrow \infty}} x^{-1} D(x, \alpha)$, where $\alpha \in$ $(\sqrt{3 / 5}, \sqrt{6})$, and $D(x, \alpha)$ is the number of times $s(n) \leq \alpha \sqrt{n}$ for $1 \leq n \leq x$.

In the positive direction, we prove that the logarithmic distribution function for $\{s(n) / \sqrt{n}\}$, defined to be

$$
\lim _{x \rightarrow \infty} \frac{1}{\log x} \sum_{\substack{1 \leq r \leq x \\ s(r) \leq \alpha \sqrt{r}}} \frac{1}{r}=L(\alpha)
$$

does exist for all $\alpha \in[\sqrt{3 / 5}, \sqrt{6}]$. We show that

$$
L(\alpha)=\frac{1}{\log 4} \int_{E_{\alpha}} \frac{1}{x} d x
$$

where the integral is a Lebesgue integral and $E_{\alpha}$ is the set $E_{\alpha}=\{x$ : $1 \leq x \leq 4$ and $\lambda(x) \leq \alpha\}$. In other words, $L(\alpha)$ is simply the (multiplicative) Haar measure of the set $E_{\alpha}$. There are similar results for $\{t(n) / \sqrt{n}\}$.

We would like to thank Igor Mikolič-Torreira for carrying out the computations in Table 1 (§6), and Richard Blecksmith for providing us with the graphs in Figure $1(\S 4)$. We are also grateful to A. J. E. M. Janssen for his remarks concering several of our proofs.

2. The functions $\lambda(x)$ and $\mu(x)$. We first prove the existence of the limit

$$
\lambda(x)=\lim _{k \rightarrow \infty} \frac{s\left(4^{k} x\right)}{\sqrt{4^{k} x}}, \quad x>0
$$

where $s(x)$ is defined in (1.1). We will require the following formulas from [1] (see Satz 3), all of which hold for integers $n \geq 0$ :

$$
\begin{cases}s(4 n)=2 s(n)-a(n), & s(4 n+2)=2 s(n)+(-1)^{n} a(n) \\ s(4 n+1)=2 s(n), & s(4 n+3)=2 s(n)\end{cases}
$$


We set $\rho(d)=\chi(1-d)$, where $\chi$ is the nontrivial character $(\bmod 4)$, so that

$$
\rho(d)=\left\{\begin{aligned}
1, & \text { if } d \equiv 0(\bmod 4), \\
-1, & \text { if } d \equiv 2(\bmod 4) \\
0, & \text { if } d \text { is odd }
\end{aligned}\right.
$$

Then, using (1.3), the relations in (2.2) can be written as the single formula (2.4) $s(4 n+d)=2 s(n)-\rho(d) a(4 n+d), \quad n \geq 0,0 \leq d \leq 3$.

We will also need the 4-adic expansion of a non-negative real number $x$, namely

$$
x=\sum_{r=0}^{\infty} d_{r} 4^{-r}
$$

where the $d_{r}$ are integers, $0 \leq d_{r} \leq 3$ for $r \geq 1$, and infinitely many $d_{r}$ are not equal to 3 . We set

$$
b_{k}=\left[4^{k} x\right]=\sum_{r=0}^{k} d_{r} 4^{k-r}
$$

and note that

$$
b_{k}=4 b_{k-1}+d_{k}, \quad \text { for } k \geq 1 \text {. }
$$

THEOREM 1. The limit in (2.1) exists for all $x>0$, and is given by the formula

$$
\lambda(x)=\frac{s(x)}{\sqrt{x}}-\frac{1}{\sqrt{x}} \sum_{r=1}^{\infty} \rho\left(d_{r}\right) a\left(b_{r}\right) 2^{-r} .
$$

Proof. We have from (2.6), (2.7), and (2.4) that

$$
\begin{aligned}
s\left(4^{k} x\right) & =s\left(\left[4^{k} x\right]\right)=s\left(b_{k}\right)=s\left(4 b_{k-1}+d_{k}\right) \\
& =2 s\left(b_{k-1}\right)-\rho\left(d_{k}\right) a\left(b_{k}\right) \\
& =2 s\left(4^{k-1} x\right)-\rho\left(d_{k}\right) a\left(b_{k}\right),
\end{aligned}
$$

for $k \geq 1$. Continuing this reduction gives

$$
s\left(4^{k} x\right)=2^{k} s(x)-\sum_{r=1}^{k} \rho\left(d_{r}\right) a\left(b_{r}\right) 2^{k-r}, \quad \text { for } k \geq 1 .
$$

Hence

$$
\frac{s\left(4^{k} x\right)}{\sqrt{4^{k} x}}=\frac{s(x)}{\sqrt{x}}-\frac{1}{\sqrt{x}} \sum_{r=1}^{k} \rho\left(d_{r}\right) a\left(b_{r}\right) 2^{-r} .
$$


Equation (2.8) now follows by letting $k \rightarrow \infty$, since the series on the right side of (2.8) converges absolutely.

COROLlaRY. If $n$ is a positive integer, then

$$
\lambda(n)=\frac{s(n-1)}{\sqrt{n}} .
$$

Proof. In the notation of (2.5) and (2.6) we have that $x=d_{0}=n$, $d_{r}=0$ for $r \geq 1$, and $b_{k}=4^{k} n$. Thus the infinite sum in (2.8) becomes

$$
\sum_{r=1}^{\infty} \rho\left(d_{r}\right) a\left(b_{r}\right) 2^{-r}=\sum_{r=1}^{\infty} a(n) 2^{-r}=a(n),
$$

and so

$$
\lambda(n)=\frac{s(n)}{\sqrt{n}}-\frac{a(n)}{\sqrt{n}}=\frac{s(n-1)}{\sqrt{n}} .
$$

Equation (2.11) suggests the following extension of the function $a(n)$.

DeFinition. For $x \geq 0$, set $x=\sum_{r=0}^{\infty} d_{r} 4^{-r}$ as in (2.5), and define

$$
a(x)=\sum_{r=1}^{\infty} \rho\left(d_{r}\right) a\left(b_{r}\right) 2^{-r},
$$

where $b_{r}=\left[4^{r} x\right]$ and $\rho(d)$ is given by (2.3).

Using (2.12), we may now write (2.8) in the form

$$
\lambda(x)=\{s(x)-a(x)\} x^{-1 / 2}, \quad x>0 .
$$

We also note the functional equation

$$
\lambda(4 x)=\lambda(x), \quad x>0,
$$

which is an immediate consequence of (2.1).

LEMMA 1. For $k \geq 0$ and $x>0$ we have the estimate

$$
\left|\lambda(x)-\frac{s\left(4^{k} x\right)}{\sqrt{4^{k} x}}\right| \leq 2^{-k} x^{-1 / 2} .
$$

Proof. It is clear from (2.12) that $|a(x)| \leq 1$.Thus, (2.13) implies

$$
\left|\lambda(x)-\frac{s(x)}{\sqrt{x}}\right| \leq x^{-1 / 2} \text {. }
$$


The lemma now follows on replacing $x$ by $4^{k} x$ and using (2.14).

LEMMA 2. (a) If $x>0$, then $\lambda(x) \in[\sqrt{3 / 5}, \sqrt{6}]$.

(b) The set $\{\lambda(x): x>0\}$ is dense in $[\sqrt{3 / 5}, \sqrt{6}]$.

Proof. For each $x_{1} \geq 1$, equation (1.5) implies the inequalities

$$
\begin{aligned}
\sqrt{\frac{3}{5}}-x_{1}^{-1 / 2} & <\frac{s\left(\left[x_{1}\right]+1\right)}{\sqrt{\left[x_{1}\right]+1}-\frac{1}{\sqrt{x_{1}}}} \\
& <\frac{s\left(\left[x_{1}\right]+1\right)-a\left(\left[x_{1}\right]+1\right)}{\sqrt{x_{1}}}=\frac{s\left(x_{1}\right)}{\sqrt{x_{1}}},
\end{aligned}
$$

and

$$
\frac{s\left(x_{1}\right)}{\sqrt{x_{1}}} \leq \frac{s\left(\left[x_{1}\right]\right)}{\sqrt{\left[x_{1}\right]}}<\sqrt{6} .
$$

Now take $x_{1}=4^{k} x$, where $k$ is chosen large enough so that $x_{1} \geq 1$. Then the above estimates give

$$
\sqrt{\frac{3}{5}}-2^{-k} x^{-1 / 2}<\frac{s\left(4^{k} x\right)}{\sqrt{4^{k} x}}<\sqrt{6}
$$

and letting $k \rightarrow \infty$ proves (a).

We also note from (2.10) that $\lambda(n)=s(n) / \sqrt{n}+o(1)$. Thus (b) follows from the fact that the set $\{s(n) / \sqrt{n}: n \geq 1\}$ is dense in $[\sqrt{3 / 5}, \sqrt{6}]$.

EXAMPLE. Let $x=(3 n+2) / 3$, where $n$ is an integer $\geq 0$. Then we have the expansion

$$
x=n+\frac{2}{3}=n+\sum_{r=1}^{\infty} \frac{2}{4^{r}},
$$

so $d_{0}=n, d_{r}=2$ and $b_{k}=4^{k} n+\sum_{r=0}^{k-1} 2 \cdot 4^{r}$ in the notation of (2.5) and (2.6). Using (1.4) it is easy to see that $a\left(b_{k}\right)=(-1)^{n} a(n)$ for all $k \geq 1$. Thus (2.12) and (2.3) imply that

$$
a(x)=\sum_{r=1}^{\infty} \rho(2) a\left(b_{r}\right) 2^{-r}=(-1)^{n+1} a(n),
$$

so from (2.13),

$$
\lambda\left(\frac{3 n+2}{3}\right)=\left[s(n)+(-1)^{n} a(n)\right]\left(\frac{3}{3 n+2}\right)^{1 / 2} .
$$


In particular,

(2.16) $\lambda\left(\frac{2}{3}\right)=2 \sqrt{\frac{3}{2}}=\sqrt{6}$ and $\lambda\left(\frac{5}{3}\right)=(2-1) \sqrt{\frac{3}{5}}=\sqrt{\frac{3}{5}}$.

We now investigate the limit

$$
\mu(x)=\lim _{k \rightarrow \infty} \frac{t\left(4^{k} x\right)}{\sqrt{4^{k} x}}, \quad x>0,
$$

where $t(x)$ is defined in (1.2). For this we recall the elementary formula

$$
t(n)=s(2 n+1)-s(n), \quad n \geq 0,
$$

from [1] (Satz 2).

THEOREM 2. The limit in (2.17) exists for all $x>0$. We have

$$
\mu(x)=\sqrt{2} \lambda(2 x)-\lambda(x),
$$

and

$$
\mu(4 x)=\mu(x) .
$$

Proof. From (2.18) it follows easily that

$$
|t(x)-s(2 x)+s(x)| \leq 1 \quad \text { for } x \geq 0 .
$$

Hence for any $x>0$,

$$
\begin{aligned}
\mu(x) & =\lim _{k \rightarrow \infty} \frac{t\left(4^{k} x\right)}{\sqrt{4^{k} x}}=\lim _{k \rightarrow \infty} \frac{s\left(2 \cdot 4^{k} x\right)-s\left(4^{k} x\right)+O(1)}{\sqrt{4^{k} x}} \\
& =\sqrt{2} \lambda(2 x)-\lambda(x) .
\end{aligned}
$$

Equation (2.20) follows immediately from (2.17).

COROLlaRY 1. For $x>0$,

$$
\lambda(x)=\sqrt{2} \mu(2 x)+\mu(x) .
$$

Proof. Equations (2.19) and (2.14) imply that

$$
\mu(2 x)=\sqrt{2} \lambda(x)-\lambda(2 x) .
$$

Multiplying through by $\sqrt{2}$ and adding to (2.19) yields the result.

COROLlaRY 2. If $n$ is a positive integer, $\mu(n)=t(n-1) / \sqrt{n}$.

Proof. Immediate from (2.19), (2.10), and (2.18). 
By virtue of (2.19), the function $\mu(x)$ inherits its properties from $\lambda(x)$. In particular, we have

Lemma 3. For $k \geq 0$ and $x>0$,

$$
\left|\mu(x)-\frac{t\left(4^{k} x\right)}{\sqrt{4^{k} x}}\right| \leq 3 \cdot 2^{-k} x^{-1 / 2} .
$$

Proof. We see from (2.19), (2.21) and Lemma 1 (with $k=0$ ) that

$$
\begin{aligned}
& \left|\frac{t(x)}{\sqrt{x}}-\mu(x)\right| \\
& \quad=\left|\frac{t(x)-s(2 x)+s(x)}{\sqrt{x}}+\frac{s(2 x)}{\sqrt{x}}-\sqrt{2} \lambda(2 x)+\lambda(x)-\frac{s(x)}{\sqrt{x}}\right| \\
& \quad \leq 3 x^{-1 / 2} .
\end{aligned}
$$

The assertion (2.22) is therefore a consequence of this estimate and (2.20).

Just as in Lemma 2, one may use (2.17), (1.6), and Corollary 2 of Theorem 2 to prove

Lemma 4. (a) If $x>0$, then $\mu(x) \in[0, \sqrt{3}]$.

(b) The set $\{\mu(x): x>0\}$ is dense in $[0, \sqrt{3}]$.

EXAMPLE. If $x=(3 n+1) / 3$, then the expansion

$$
x=n+\frac{1}{3}=n+\sum_{r=1}^{\infty} \frac{1}{4^{r}}
$$

implies by (2.3) and (2.12) that $a(x)=0$. Hence

$$
\lambda\left(\frac{3 n+1}{3}\right)=s(n)\left(\frac{3}{3 n+1}\right)^{1 / 2} .
$$

It follows from this and equations (2.19), (2.16) that

$$
\mu\left(\frac{1}{3}\right)=\sqrt{3}, \quad \mu\left(\frac{2}{3}\right)=0 .
$$

The examples of this section suggest that $\sqrt{x} \lambda(x)$ is a rational number whenever $x$ is. This is indeed true, as we shall now show.

TheOrem 3. If $x>0$ and $x \in \mathbf{Q}$, then $\sqrt{x} \lambda(x) \in \mathbf{Q}$. 
Proof. By (2.13) it suffices to show that $a(x) \in \mathbf{Q}$ if $x \in \mathbf{Q}$, since $s(x) \in \mathbf{Z}$. If $x$ is rational, the 4-adic expansion of $x$ must be ultimately periodic:

$$
x=\sum_{r=0}^{\infty} d_{r} 4^{-r} \text { where } d_{k+p}=d_{k}, k \geq k_{0},
$$

for some period length $p$ and some $k_{0} \geq 1$. To prove that $a(x) \in \mathbf{Q}$ it is enough to prove that

$$
\rho\left(d_{k+2 p}\right) a\left(b_{k+2 p}\right)=\rho\left(d_{k}\right) a\left(b_{k}\right), \quad k \geq k_{0},
$$

by formula (2.12). Clearly $\rho\left(d_{k+2 p}\right)=\rho\left(d_{k}\right), k \geq k_{0}$, and so we consider the term $a\left(b_{k+2 p}\right)$.

From (2.6) we have

$$
b_{k+p}=4^{p} b_{k}+\sum_{r=1}^{p} d_{k+r^{4}} 4^{p-r}=4^{p} b_{k}+b_{k}^{\prime} .
$$

We first compute $a\left(b_{k+p}\right)$ using (1.4), in which $e(n)$ is the number of pairs of consecutive ones in the binary representation of $n$. Now the binary representation of $b_{k+p}$ is pieced together from the binary representations of $b_{k}$ and $b_{k}^{\prime}$, by (2.24). Moreover, a 1 occurs simultaneously in the last binary digit of $b_{k}$ and the first binary digit of $b_{k}^{\prime}$ if and only if $2 \nmid d_{k}$ and $d_{k+1}=2$ or 3 . Thus we have

$$
\begin{aligned}
a\left(b_{k+p}\right) & =a\left(b_{k}\right) a\left(b_{k}^{\prime}\right)(-1)^{d_{k}\left[d_{k+1} / 2\right]} \\
& =a\left(b_{k}\right) \varepsilon_{k}, \quad \text { for } k \geq k_{0},
\end{aligned}
$$

where $\varepsilon_{k}= \pm 1$. Since $b_{k+p}^{\prime}=b_{k}^{\prime}, \varepsilon_{k+p}=\varepsilon_{k}$ for $k \geq k_{0}$; we deduce that

$$
a\left(b_{k+2 p}\right)=a\left(b_{k+p}\right) \varepsilon_{k} \varepsilon_{k+p}=a\left(b_{k}\right),
$$

and this proves (2.23).

Corollary. If $x>0$ and $x \in \mathbf{Q}$, then $\sqrt{x} \mu(x) \in \mathbf{Q}$.

Proof. This is clear from (2.19).

As a further example of Theorem 3 we note that

$$
\frac{1}{\sqrt{73}} \lambda\left(\frac{1}{73}\right)=\frac{65297}{65408}=\frac{17 \cdot 23 \cdot 167}{2^{7} \cdot 7 \cdot 73},
$$

where the value

$$
a\left(\frac{1}{73}\right)=\frac{111}{65408}=\frac{3 \cdot 37}{2^{7} \cdot 7 \cdot 73}
$$


is readily obtained from 4-adic expansion

$$
\frac{1}{73}=\overline{.000320013} \text {. }
$$

We remark that the converse of Theorem 3 is certainly false, since there are irrational numbers $x=\sum_{r=0}^{\infty} d_{r} 4^{-r}$ for which $d_{r}$ is always odd; for these $x$ we have $a(x)=0$ from (2.3), so $\sqrt{x} \lambda(x)=s(x) \in \mathbf{Z}$.

3. The continuity of $\lambda(x)$ and $\mu(x)$. In this section we show that $\lambda(x)$ and $\mu(x)$ are actually continuous functions of $x$, for $x>0$. Equation (2.19) shows that it is enough to prove this for $\lambda(x)$.

We first consider the function $a(x)$.

THEOREM 4. Let $x_{0}>0$. Then $a(x)$ is continuous at $x_{0}$ if and only if $x_{0}$ is not a natural number. If $x_{0}$ is a natural number, then

$$
\lim _{x \rightarrow x_{0}^{-}} a(x)=0 \text { and } \lim _{x \rightarrow x_{0}^{+}} a(x)=a\left(x_{0}\right)= \pm 1 .
$$

Proof. We prove the theorem in three parts:

(i) $a(x)$ is continuous from the right at any $x_{0}>0$;

(ii) $a(x)$ is continuous from the left at $x_{0} \notin \mathbf{N}$;

(iii) $\lim _{x \rightarrow x_{0}^{-}} a(x)=0$, if $x_{0} \in \mathbf{N}$.

Here $\mathbf{N}$ denotes the set of natural numbers.

(i) Assume $x_{0}=\sum_{r=0}^{\infty} d_{r} 4^{-r}$ as in (2.5), and define $x_{n}$ by $4^{n} x_{n}=\left[4^{n} x_{0}\right]$ $+1=\dot{b}_{n}+1$, for $n \geq 1$, so that $x_{n}>x_{0}$ and $x_{n} \rightarrow x_{0}$ as $n \rightarrow \infty$. If $x_{0}<x^{*}<x_{n}$, then $x^{*}=\sum_{r=0}^{\infty} d_{r}^{*} 4^{-r}$ with $d_{r}^{*}=d_{r}$ for $0 \leq r \leq n$. Hence, by $(2.6), b_{r}^{*}=\left[4^{r} x^{*}\right]=\left[4^{r} x_{0}\right]=b_{r}$ for $0 \leq r \leq n$, and by (2.12) we have that

$$
\begin{aligned}
\mid a\left(x_{0}\right)- & a\left(x^{*}\right)|=| \sum_{r=1}^{\infty} \rho\left(d_{r}\right) a\left(b_{r}\right) 2^{-r}-\sum_{r=1}^{\infty} \rho\left(d_{r}^{*}\right) a\left(b_{r}^{*}\right) 2^{-r} \mid \\
& \leq \sum_{r=n+1}^{\infty}\left|\rho\left(d_{r}\right) a\left(b_{r}\right)-\rho\left(d_{r}^{*}\right) a\left(b_{r}^{*}\right)\right| 2^{-r} \leq \sum_{r=n+1}^{\infty} 2^{1-r}=2^{1-n} .
\end{aligned}
$$

This clearly implies (i).

(ii) Here there are two cases:

(a) If $x_{0}=\sum_{r=0}^{\infty} d_{r} 4^{-r}$, where infinitely many $d_{r}$ are nonzero, then we set $x_{n}=\sum_{r=0}^{n} d_{r} 4^{-r}$, so that $x_{n}<x_{0}$ and $x_{n} \rightarrow x_{0}$ as $n \rightarrow \infty$. If $x^{*}$ satisfies $x_{n}<x^{*}<x_{0}$, then clearly $x^{*}=\sum_{r=0}^{\infty} d_{r}^{*} 4^{-r}$ with $d_{r}^{*}=d_{r}$ for $0 \leq r \leq n$, and as in (i) we find that $\left|a\left(x_{0}\right)-a\left(x^{*}\right)\right| \leq 2^{1-n}$. 
(b) In the second case, $x_{0}=\sum_{r=0}^{s} d_{r} 4^{-r}$, where $s \geq 1$ and $d_{s} \neq 0$. Let $n \geq s+1$ and define

$$
x_{n}=x_{0}-4^{-n}=\sum_{r=0}^{s-1} d_{r} 4^{-r}+\frac{d_{s}-1}{4^{s}}+\sum_{r=s+1}^{n} 3 \cdot 4^{-r} .
$$

For any $x^{*}$ in the interval $x_{n}<x^{*}<x_{0}$, we then have $x^{*}=\sum_{r=0}^{\infty} d_{r}^{*} 4^{-r}$, with

$$
d_{r}^{*}= \begin{cases}d_{r}, & \text { for } 0 \leq r \leq s-1, \\ d_{s}-1, & \text { for } r=s, \\ 3, & \text { for } s+1 \leq r \leq n .\end{cases}
$$

Thus, we see from (2.12) that

$$
\begin{aligned}
a\left(x^{*}\right) & =\sum_{r=1}^{n} \rho\left(d_{r}^{*}\right) a\left(b_{r}^{*}\right) 2^{-r}+\sum_{r=n+1}^{\infty} \rho\left(d_{r}^{*}\right) a\left(b_{r}^{*}\right) 2^{-r} \\
& =\sum_{r=1}^{s-1} \rho\left(d_{r}\right) a\left(b_{r}\right) 2^{-r}+\rho\left(d_{s}-1\right) a\left(b_{s}^{*}\right) 2^{-s}+\sum_{r=n+1}^{\infty} \rho\left(d_{r}^{*}\right) a\left(b_{r}^{*}\right) 2^{-r} \\
& =\sum_{r=1}^{s-1} \rho\left(d_{r}\right) a\left(b_{r}\right) 2^{-r}+\rho\left(d_{s}-1\right) a\left(b_{s}-1\right) 2^{-s}+O\left(2^{-n}\right),
\end{aligned}
$$

since $b_{s}^{*}=4 b_{s-1}^{*}+d_{s}^{*}=4 b_{s-1}+d_{s}-1=b_{s}-1$. On the other hand,

$$
\begin{aligned}
a\left(x_{0}\right) & =\sum_{r=1}^{s} \rho\left(d_{r}\right) a\left(b_{r}\right) 2^{-r}+\sum_{r=s+1}^{\infty} \rho(0) a\left(b_{r}\right) 2^{-r} \\
& =\sum_{r=1}^{s-1} \rho\left(d_{r}\right) a\left(b_{r}\right) 2^{-r}+\rho\left(d_{s}\right) a\left(b_{s}\right) 2^{-s}+a\left(b_{s}\right) \sum_{r=s+1}^{\infty} 2^{-r} \\
& =\sum_{r=1}^{s-1} \rho\left(d_{r}\right) a\left(b_{r}\right) 2^{-r}+\rho\left(d_{s}\right) a\left(b_{s}\right) 2^{-s}+a\left(b_{s}\right) 2^{-s},
\end{aligned}
$$

and subtracting the expressions for $a\left(x^{*}\right)$ and $a\left(x_{0}\right)$ gives

$$
\begin{aligned}
a\left(x^{*}\right)- & a\left(x_{0}\right) \\
= & {\left[\rho\left(d_{s}-1\right) a\left(b_{s}-1\right)-\rho\left(d_{s}\right) a\left(b_{s}\right)-a\left(b_{s}\right)\right] 2^{-s}+O\left(2^{-n}\right) . }
\end{aligned}
$$

We now claim that the expression $E_{s}$ inside the brackets is zero. To show this we must consider the three possibilities: $d_{s}=1,2$, or 3 (note $d_{s} \neq 0$ by assumption). Recall that $b_{s}=4 b_{s-1}+d_{s}$.

If $d_{s}=1$, then

$$
\begin{aligned}
E_{s} & =\rho(0) a\left(b_{s}-1\right)-a\left(b_{s}\right)=a\left(4 b_{s-1}\right)-a\left(4 b_{s-1}+1\right) \\
& =a\left(b_{s-1}\right)-a\left(b_{s-1}\right)=0, \quad \text { by }(1.3) .
\end{aligned}
$$


If $d_{s}=2$, then $E_{s}=-\rho(2) a\left(b_{s}\right)-a\left(b_{s}\right)=0$.

If $d_{s}=3$, then $E_{s}=\rho(2) a\left(b_{s}-1\right)-a\left(b_{s}\right)=-a\left(4 b_{s-1}+2\right)-$ $a\left(4 b_{s-1}+3\right)=-a\left(2 b_{s-1}+1\right)+a\left(2 b_{s-1}+1\right)=0$, again by (1.3).

Thus, we have that $\left|a\left(x^{*}\right)-a\left(x_{0}\right)\right|=O\left(2^{-n}\right)$, when $x_{n}<x^{*}<x_{0}$, for any $n \geq s+1$, and this shows that $a(x)$ is continuous from the left at $x_{0}$.

(iii) Assume now that $x_{0} \in \mathbf{N}$, and define

$$
x_{n}=x_{0}-4^{-n}=x_{0}-1+\sum_{r=1}^{n} 3 \cdot 4^{-r}, \quad n \geq 1 .
$$

As in (ii) we have for any $x^{*}$ in the interval $x_{n}<x^{*}<x_{0}$ that $x^{*}=$ $\sum_{r=0}^{\infty} d_{r}^{*} 4^{-r}$, where

$$
d_{r}^{*}= \begin{cases}x_{0}-1, & \text { for } r=0, \\ 3, & \text { for } 1 \leq r \leq n .\end{cases}
$$

Hence, $a\left(x^{*}\right)=\sum_{r=1}^{n} \rho(3) a\left(b_{r}^{*}\right) 2^{-r}+O\left(2^{-n}\right)=O\left(2^{-n}\right)$, since $\rho(3)=0$. But this implies $a\left(x^{*}\right) \rightarrow 0$ as $x^{*} \rightarrow x_{0}$ from below.

REMARK. The same proof shows that the complex valued function

$$
a_{\tau}(x)=\sum_{r=1}^{\infty} \rho\left(d_{r}\right) a\left(b_{r}\right) 2^{-\tau r},
$$

defined for complex numbers $\tau$ with positive real part, is continuous at $x_{0}$ whenever $x_{0} \notin \mathbf{N}$, and that

$$
\lim _{x \rightarrow x_{0}^{-}} a_{\tau}(x)=0, \quad \lim _{x \rightarrow x_{0}^{+}} a_{\tau}(x)=a_{\tau}\left(x_{0}\right), \quad \text { if } x_{0} \in \mathbf{N} .
$$

THEOREM 5. $\lambda(x)$ is continuous for $x>0$.

Proof. Let $x_{0}>0$. If $x_{0} \notin \mathbf{N}$, then it follows from Theorem 4, equation (2.13), and the fact that $s(x)$ is a step-function that $\lambda(x)$ is continuous at $x_{0}$. If $x_{0} \in \mathbf{N}$, the same considerations show that $\lambda(x)$ is continuous from the right at $x_{0}$. Furthermore, by (2.13), (3.1), and (2.10) we have that

$$
\begin{aligned}
\lim _{x^{*} \rightarrow x_{0}^{-}} \lambda\left(x^{*}\right) & =\lim _{x^{*} \rightarrow x_{0}^{-}}\left[s\left(x_{0}-1\right)-a\left(x^{*}\right)\right]\left(x^{*}\right)^{-1 / 2} \\
& =\frac{s\left(x_{0}-1\right)}{\sqrt{x_{0}}}=\lambda\left(x_{0}\right) .
\end{aligned}
$$

Therefore $\lambda(x)$ is continuous at $x_{0}$. 
COROLlaRY 1. The function $\lambda(x)$ maps both intervals $(0, \infty)$ and $[1,4]$ continuously onto $[\sqrt{3 / 5}, \sqrt{6}]$.

Proof. This is immediate from Theorem 5, (2.14) and Lemma 2. Alternatively, one may deduce Corollary 1 from the intermediate value theorem and the values $\lambda(5 / 3)=\sqrt{3 / 5}, \lambda(8 / 3)=\sqrt{6}$.

Corollary 2. The function $\mu(x)$ maps $(0, \infty)$ and $[1,4]$ continuously onto $[0, \sqrt{3}]$.

We remark that the continuity of $\lambda(x)$ for $x>0$ also follows from the fact that the functions $f_{k}(x)=s\left(4^{k} x\right)\left(4^{k} x\right)^{-1 / 2}$ converge uniformly to $\lambda(x)$ on any interval $[a, b]$ with $0<a<b$, by (2.15). The functions $f_{k}(x)$ are step functions with jump discontinuities of order $2^{-k} x^{-1 / 2}$ at the points $x$ for which $4^{k} x \in \mathbf{N}$. The continuity of $\lambda(x)$ may then be deduced from the following general result, whose proof we leave to the reader.

THEOREM. Let $J$ be an interval, and let $\left\{f_{k}(x)\right\}$ be a sequence of functions converging uniformly to $f(x)$ on $J$. Assume for every $x_{0}$ in $J$ that

$$
d_{k}\left(x_{0}\right)=\limsup _{x \rightarrow x_{0}}\left|f_{k}(x)-f_{k}\left(x_{0}\right)\right| \rightarrow 0, \text { as } k \rightarrow \infty .
$$

Then $f(x)$ is continuous on $J$.

4. The non-differentiability of $\lambda(x)$. Although $\lambda(x)$ is a continuous function, it is differentiable almost nowhere. To prove this we first recall the following definition. (See [6], Ch. 8.)

Definition. A real number $x_{0}>0$ is normal (to the base 4) if and only if the numbers $x_{0}, 4 x_{0}, 4^{2} x_{0}, \ldots, 4^{n} x_{0}, \ldots$ are uniformly distributed modulo 1.

An equivalent definition is the following. Let $k \geq 1$, and let $B_{k}$ be a block of $k$ digits to the base 4. Also let $x_{0}=\sum_{r=0}^{\infty} d_{r} 4^{-r}$, and denote by $N\left(m, B_{k}\right)$ the number of occurrences of the block $B_{k}$ in the initial block .$d_{1} d_{2} \ldots d_{m}$ of $x_{0}-d_{0}$. (For example, if $x_{0}=.1121121102$ and $B_{5}=11211$, we have $N\left(10, B_{5}\right)=2$.) Then $x_{0}$ is normal if and only if

$$
\lim _{m \rightarrow \infty} \frac{1}{m} N\left(m, B_{k}\right)=4^{-k}
$$

for all $k \geq 1$ and all blocks $B_{k}$ of length $k$. 
It is well-known [6] that almost all positive real numbers are normal. In particular, almost all positive real numbers $x_{0}=\sum_{r=0}^{\infty} d_{r} 4^{-r}$ have the property that $d_{n}=d_{n+1}=0$ for infinitely many $n$. This is the essential fact we use in proving

THEOREM 6. If $x_{0}>0$ is normal (to the base 4), then $\lambda(x)$ is not differentiable at $x_{0}$. Thus, $\lambda(x)$ is non-differentiable almost everywhere.

Proof. Since $\sqrt{x} \lambda(x)=s(x)-a(x)$, it is enough to prove that

$$
\frac{1}{h}\left\{a\left(x_{0}+h\right)-a\left(x_{0}\right)\right\}
$$

is unbounded as $h \rightarrow 0^{+}$. The theorem then follows from the fact that the step function $s(x)$ has right derivative 0 for all $x_{0}>0$.

So let $x_{0}=\sum_{r=0}^{\infty} d_{r} 4^{-r}$, choose an $n \geq 1$ for which $d_{n}=d_{n+1}=0$, and set $h=4^{-n}$. Then the 4-adic expansion of $x_{0}+h$ is

$$
x_{0}+h=\sum_{r=0}^{n-1} d_{r^{4}} 4^{-r}+4^{-n}+\sum_{r=n+1}^{\infty} d_{r} 4^{-r} .
$$

Putting $b_{r}^{\prime}=\left[4^{r}\left(x_{0}+4^{-n}\right)\right]$, we have $b_{r}^{\prime}=b_{r}$ for $r \leq n-1$, while $b_{n}^{\prime}=$ $4 b_{n-1}+1=b_{n}+1$ and $b_{n+1}^{\prime}=4 b_{n}^{\prime}=b_{n+1}+4$. Thus (1.3) implies $a\left(b_{n}^{\prime}\right)$ $=a\left(b_{n-1}\right)=a\left(b_{n}\right)$ and $a\left(b_{n+1}^{\prime}\right)=a\left(b_{n}^{\prime}\right)=a\left(b_{n}\right)=a\left(b_{n+1}\right)$. Furthermore, using (1.4) and considering the binary expansions of $b_{m}^{\prime}$ and $b_{m}$, we see that $a\left(b_{m}^{\prime}\right)=a\left(b_{m}\right)$, for $m \geq n+2$. Hence

$$
\begin{aligned}
a\left(x_{0}+4^{-n}\right)-a\left(x_{0}\right)= & \sum_{r=1}^{n-1} \frac{\rho\left(d_{r}\right) a\left(b_{r}\right)}{2^{r}}+\sum_{r=n+1}^{\infty} \frac{\rho\left(d_{r}\right) a\left(b_{r}^{\prime}\right)}{2^{r}} \\
& -\sum_{r=1}^{\infty} \frac{\rho\left(d_{r}\right) a\left(b_{r}\right)}{2^{r}}=-a\left(b_{n}\right) 2^{-n},
\end{aligned}
$$

and so

$$
4^{n}\left\{a\left(x_{0}+4^{-n}\right)-a\left(x_{0}\right)\right\}=-a\left(b_{n}\right) 2^{n}= \pm 2^{n} .
$$

Since there are infinitely many $n$ for which $d_{n}=d_{n+1}=0$, this proves that the expression (4.2) is indeed unbounded as $h \rightarrow 0^{+}$.

We remark that the same proof shows $\lambda(x)$ is not differentiable at any positive rational $x_{0}$ whose denominator is a power of 2 .

The proof of Theorem 6 can also be modified to show that for a normal number $x_{0}$, the quotient (4.2) takes on all real values infinitely often as $h \rightarrow 0^{+}$. For one can choose a sequence $n_{k}$ with $d_{n_{k}}=d_{n_{k}+1}=0$, 
$k \geq 1$, such that $a\left(b_{n_{k}}\right)$ changes sign infinitely often. (For example, the block 00300 occurs infinitely often among the digits of $x_{0}$. If the block starts at the index $n$, and $n_{k}=n, n_{k+1}=n+3$, then $a\left(b_{n_{k+1}}\right)=-a\left(b_{n_{k}}\right)$.) It follows from (4.3) that the quotient (4.2), which is continuous in $h$ for small $h$, takes on arbitrarily large positive and negative values as $h \rightarrow 0^{+}$. The intermediate value theorem then shows the truth of the claim above. This remark is due to A. J. E. M. Janssen (private communication).

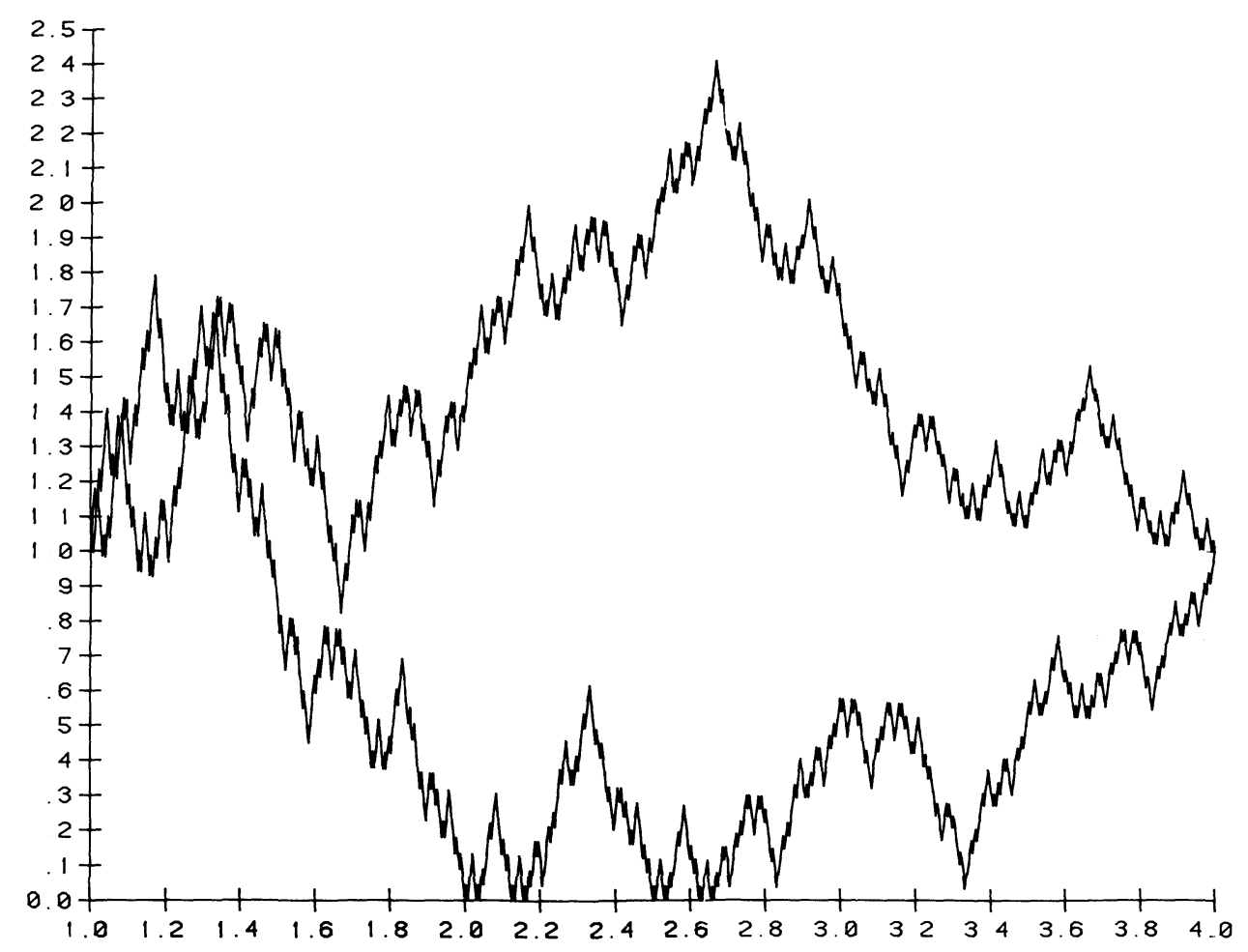

Felis Electrica

FIGURE 1. Polygonal approximations to $\lambda(x)$ and $\mu(x),(\lambda(x) \geq \mu(x)$.)

The upper graph in Figure 1 is the polygonal curve joining the points $\left(1+\frac{n}{4^{5}}, \lambda\left(1+\frac{n}{4^{5}}\right)\right)=\left(1+\frac{n}{4^{5}}, \frac{s\left(4^{5}+n-1\right)}{\sqrt{4^{5}+n}}\right), n=0,1, \ldots, 3 \cdot 4^{5}$.

The lower graph is the same with the function $s$ replaced by the function $t$, and $\lambda$ replaced by $\mu$. 
5. The Fourier series of $\lambda(x)$. It follows from the continuity of $\lambda(x)$ and (2.14) that the function

$$
f(x)=\lambda\left(4^{x / 2 \pi}\right)
$$

is continuous for all $x$ and has period $2 \pi$. Thus $f$ has a Fourier series

$$
f(x)=\sum_{n=-\infty}^{\infty} c_{n} e^{i n x}, \quad c_{n}=\frac{1}{2 \pi} \int_{0}^{2 \pi} f(\theta) e^{-i n \theta} d \theta
$$

which is $(C, 1)$ summable to $f(x)$ for all $x$. (See [3], p. 62) Using $\lambda(x)=f(\pi \log x / \log 2)$, this easily yields the following result for $\lambda(x)$.

THEOREM 7. The function $\lambda(x)$ has the logarithmic Fourier series expansion

$$
\lambda(x)=\sum_{n=-\infty}^{\infty} c_{n} e^{\pi i n \log x / \log 2}, \quad x>0
$$

where

$$
c_{n}=\frac{1}{\log 4} \int_{1}^{4} \frac{\lambda(x)}{x^{1 / 2+\gamma_{n}}} d x, \quad \gamma_{n}=\frac{1}{2}+\frac{\pi n i}{\log 2},
$$

and where the infinite series converges in the $(C, 1)$ sense for all $x>0$, i.e.

$$
\sum_{n=-\infty}^{\infty} c_{n} e^{\pi i n \log x / \log 2}=\lim _{k \rightarrow \infty} \frac{1}{k+1}\left(\sigma_{0}+\sigma_{1}+\cdots+\sigma_{k}\right),
$$

with

$$
\sigma_{k}=\sum_{n=-k}^{k} c_{n} e^{\pi i n \log x / \log 2}
$$

COROLlaRY. For $x>0$ we have

$$
s(x)=\sum_{n=-\infty}^{\infty} c_{n} x^{1 / 2+\pi n i / \log 2}+a(x),
$$

where the series is $(C, 1)$ summable for all $x>0$, and $c_{n}$ is defined by (5.3).

Proof. This is immediate from (5.2) and (2.13).

We note that the series in (5.2) and (5.4) are convergent in the usual sense for almost all $x>0$, by the deep theorem of Carleson [2]. However, 
it is possible to give a direct proof of this fact. We first prove

LEMMA 5. If $x_{0}>0$ is normal (to the base 4), then

$$
\left|a\left(x_{0}+h\right)-a\left(x_{0}\right)\right|=O\left(|h|^{1 / 4}\right), \quad \text { as } h \rightarrow 0,
$$

where the implied constant depends only on $x_{0}$.

Proof. Let $x_{0}=\sum_{r=0}^{\infty} d_{r} 4^{-r}$, and assume $4^{-n-1} \leq h<4^{-n}, n \geq 1$, so that

$$
h=\sum_{r=n+1}^{\infty} h_{r} 4^{-r}, \quad 0 \leq h_{r} \leq 3, h_{n+1} \neq 0 .
$$

Then

$$
x_{0}+h=\sum_{r=0}^{n} d_{r} 4^{-r}+\sum_{r=n+1}^{\infty}\left(d_{r}+h_{r}\right) 4^{-r} .
$$

Since $d_{r}$ and $h_{r}$ are digits, not all equal to 3 past some point, we have that

$$
\sum_{r=n+1}^{\infty}\left(d_{r}+h_{r}\right) 4^{-r}<\sum_{r=n+1}^{\infty} 6 \cdot 4^{-r}=2 \cdot 4^{-n},
$$

so

$$
x_{0}+h=\sum_{r=0}^{n} d_{r} 4^{-r}+\sum_{r=n}^{\infty} h_{r}^{\prime} 4^{-r},
$$

where the $h_{r}^{\prime}$ are digits and $h_{n}^{\prime}=0$ or 1 . If $h_{n}^{\prime}=1$, then there is a carry into the $n$th place in (5.6). However the carrying will stop as soon as some $d_{r} \neq 3, r \leq n$.

In order to estimate how long the carrying continues, we apply (4.1) to the number $x_{0}$ and the block $B_{1}=3$. By that equation we may choose an $n_{0}$ so that

$$
N\left(m, B_{1}\right)<\frac{3 m}{8}, \text { for } m \geq n_{0} .
$$

Therefore, if $n \geq n_{0}$, the number of digits $d_{r}$ equal to 3 between $n / 2$ and $n$ is at most $3 n / 8<n / 2$. Hence there is an $r_{0}>n / 2$ for which $d_{r_{0}} \neq 3$, and this implies that

$$
x_{0}+h=\sum_{r=0}^{r_{0}-1} d_{r} 4^{-r}+\sum_{r \geq r_{0}} d_{r}^{\prime} 4^{-r},
$$

where the $d_{r}^{\prime}$ are digits. 
Now apply (2.12) with $b_{r}^{\prime}=\left[4^{r} x_{0}+4^{r} h\right], b_{r}=\left[4^{r} x_{0}\right]$, to give

$$
\begin{aligned}
\left|a\left(x_{0}+h\right)-a\left(x_{0}\right)\right| & =\left|\sum_{r \geq r_{0}} \rho\left(d_{r}^{\prime}\right) a\left(b_{r}^{\prime}\right) 2^{-r}-\sum_{r \geq r_{0}} \rho\left(d_{r}\right) a\left(b_{r}\right) 2^{-r}\right| \\
& \leq 2 \sum_{r \geq r_{0}} 2^{-r}=\frac{4}{2^{r_{0}}}<\frac{4}{2^{n / 2}} \leq 4 \sqrt{2} h^{1 / 4},
\end{aligned}
$$

for $n \geq n_{0}$. Thus

$$
\left|a\left(x_{0}+h\right)-a\left(x_{0}\right)\right|=O\left(h^{1 / 4}\right), \quad \text { as } h \rightarrow 0^{+} .
$$

A similar discussion shows that

$$
\left|a\left(x_{0}-h\right)-a\left(x_{0}\right)\right|=O\left(h^{1 / 4}\right), \quad \text { as } h \rightarrow 0^{+},
$$

and this completes the proof of the lemma.

THEOREM 8. If $x_{0}>0$ is a normal number (to the base 4), then the Fourier series (5.2) of $\lambda(x)$ converges at $x_{0}$. Thus, (5.2) and (5.4) converge for almost all positive real numbers $x$.

Proof. Since $x_{0}$ is not an integer, $s\left(x_{0}+h\right)=s\left(x_{0}\right)$ for small $h$, and so (2.13) gives that

$$
\begin{aligned}
\lambda\left(x_{0}+h\right) & -\lambda\left(x_{0}\right)=\frac{s\left(x_{0}\right)}{\sqrt{x_{0}+h}}-\frac{s\left(x_{0}\right)}{\sqrt{x_{0}}}-\frac{a\left(x_{0}+h\right)}{\sqrt{x_{0}+h}}+\frac{a\left(x_{0}\right)}{\sqrt{x_{0}}} \\
& =\frac{s\left(x_{0}\right)}{\sqrt{x_{0}+h}}\left(1-\sqrt{1+\frac{h}{x_{0}}}\right)-\frac{a\left(x_{0}+h\right)-a\left(x_{0}\right)}{\sqrt{x_{0}+h}} \\
& +\frac{a\left(x_{0}\right)}{\sqrt{x_{0}+h}}\left(\sqrt{1+\frac{h}{x_{0}}}-1\right) .
\end{aligned}
$$

Now $\left(x_{0}+h\right)^{-1 / 2}$ is bounded as $h \rightarrow 0$, and $\left(1+h / x_{0}\right)^{1 / 2}=1+h / 2 x_{0}$ $+O\left(h^{2}\right)=1+O\left(|h|^{1 / 4}\right)$, as $h \rightarrow 0$. Therefore, Lemma 5 implies that

$$
\left|\lambda\left(x_{0}+h\right)-\lambda\left(x_{0}\right)\right|=O\left(|h|^{1 / 4}\right), \quad \text { as } h \rightarrow 0 .
$$

We set $y=1+h / x_{0}$, and use the fact that $h \simeq x_{0} \log y$ as $h \rightarrow 0$ to write the last estimate in the form

$$
\left|\lambda\left(x_{0} y\right)-\lambda\left(x_{0}\right)\right|=O\left(|\log y|^{1 / 4}\right), \quad \text { as } y \rightarrow 1
$$


If $z_{0}=\pi \log x_{0} / \log 2$, then this gives the following estimate for the function $f(z)=\lambda\left(4^{z / 2 \pi}\right)$ :

$$
\left|f\left(z_{0}+h\right)-f\left(z_{0}\right)\right|=O\left(|h|^{1 / 4}\right), \quad \text { as } h \rightarrow 0 .
$$

But this condition implies the convergence of the Fourier series of $f$ at $z_{0}$ (see [3], p. 41), and therefore the convergence of (5.2) at $x_{0}$.

REMARK. If we define a simply normal number to be a number $x_{0}$ which satisfies the condition (4.1) just for $k=1$, i.e. for blocks of length one, then it is clear that the conclusions of Lemma 5 and Theorem 8 hold for the larger set of simply normal numbers. Thus both (5.2) and (5.4) converge for example at the point

$$
x=m .01230123 \cdots_{4}=m+\frac{9}{85},
$$

where $m$ is a non-negative integer. Similarly, (5.2) and (5.4) converge at any point $x_{0}=\sum_{r=0}^{\infty} d_{r} 4^{-r}$ which has the property that $d_{r} \neq 0$ or 3 for large $r$, e.g. the point $x_{0}=.1212 \cdots_{4}=2 / 5$.

Our results for $\lambda(x)$ and $s(x)$ are easily extended to the functions $\mu(x)$ and $t(x)$ using (2.18) and (2.19). For example, $\mu(x)$ has the logarithmic Fourier series

$$
\mu(x)=\sum_{n=-\infty}^{\infty} c_{n}\left\{(-1)^{n} \sqrt{2}-1\right\} e^{\pi i n \log x / \log 2},
$$

which is $(C, 1)$ summable to $\mu(x)$ for all $x>0$, and which is actually convergent in case $x$ is normal to the base 4 (for then $2 x$ is also normal). Moreover, (2.18) implies easily that

$$
\begin{aligned}
t(x) & =s(2 x)-s(x)+\frac{1}{2}\left(1+(-1)^{\left[d_{1} / 2\right]}\right)(-1)^{d_{0}} a\left(b_{0}\right) \\
& =\sqrt{x} \mu(x)+b(x) \\
& =\sum_{n=-\infty}^{\infty} c_{n}\left\{(-1)^{n} \sqrt{2}-1\right\} x^{1 / 2+\pi n i / \log 2}+b(x),
\end{aligned}
$$

where

$$
b(x)=a(2 x)-a(x)+\frac{1}{2}\left(1+(-1)^{\left[d_{1} / 2\right]}\right)(-1)^{d_{0}} a\left(b_{0}\right),
$$


and $x$ is given by (2.5). The function $b(x)$ has properties analogous to those of $a(x)$. For instance, $b(n)=(-1)^{n} a(n)$, for $n \geq 0 ; b(x)$ is continuous at $x_{0}$ if $x_{0} \neq \mathbf{N}$; and

$$
\lim _{x \rightarrow x_{0}^{-}} b(x)=0, \quad \lim _{x \rightarrow x_{0}^{+}} b(x)=b\left(x_{0}\right), \quad \text { if } x_{0} \in \mathbf{N} .
$$

6. The Fourier coefficients $c_{n}$. Concerning the coefficients $c_{n}$, we first prove

THEOREM 9. Infinitely many of the coefficients $c_{n}$ are nonzero; in fact $c_{n} \neq O\left(|n|^{-2-\delta}\right)$, as $n \rightarrow \pm \infty$, for any $\delta>0$.

Proof. Assume that $c_{n}=O\left(|n|^{-2-\delta}\right)$ for some $\delta>0$. Then the series in (5.2) converges to $\lambda(x)$ for all $x>0$, and the differentiated series

$$
\frac{\pi i}{x \log 2} \sum_{n=-\infty}^{\infty} n c_{n} x^{\pi i n / \log 2}=\Delta(x)
$$

converges uniformly for $x \geq 1$. Therefore $\lambda^{\prime}(x)=\Delta(x)$ for all $x>1$, which contradicts Theorem 6. Hence $c_{n}=O\left(|n|^{-2-\delta}\right)$ is false.

We shall now relate the $c_{n}$ to the behavior of the function $\eta(\tau)$ defined by the Dirichlet series

$$
\eta(\tau)=\sum_{n=1}^{\infty} \frac{a(n)}{n^{\tau}} .
$$

By virtue of (1.5), this series converges in the half-plane $\operatorname{Re} \tau>1 / 2$, and absolutely for $\operatorname{Re} \tau>1$. (See [5], p. 123.) Using partial summation to express $\eta(\tau)$ as an integral gives

$$
\begin{aligned}
\eta(\tau) & =\sum_{n=1}^{\infty} \frac{s(n)-s(n-1)}{n^{\tau}}=-1+\sum_{n=1}^{\infty} s(n)\left\{\frac{1}{n^{\tau}}-\frac{1}{(n+1)^{\tau}}\right\} \\
& =-1+\tau \sum_{n=1}^{\infty} s(n) \int_{n}^{n+1} \frac{1}{x^{\tau+1}} d x \\
& =-1+\tau \int_{1}^{\infty} \frac{s(x)}{x^{\tau+1}} d x, \text { for } \operatorname{Re} \tau>\frac{1}{2} .
\end{aligned}
$$

We substitute $s(x)=\sqrt{x} \lambda(x)+a(x)$, and find

$$
\eta(\tau)=-1+\tau \int_{1}^{\infty} \frac{\lambda(x)}{x^{\tau+1 / 2}} d x+\tau \int_{1}^{\infty} \frac{a(x)}{x^{\tau+1}} d x .
$$


Now rearrange the first integral using (2.14):

$$
\begin{aligned}
\int_{1}^{\infty} \frac{\lambda(x)}{x^{\tau+1 / 2}} d x & =\sum_{k=0}^{\infty} \int_{4^{k}}^{4^{k+1}} \frac{\lambda(x)}{x^{\tau+1 / 2}} d x \\
& =\sum_{k=0}^{\infty} \int_{1}^{4} \frac{\lambda(x)}{2^{k(2 \tau-1)} u^{\tau+1 / 2}} d u, \\
& =\left(1-2^{1-2 \tau}\right)^{-1} \int_{1}^{4} \frac{\lambda(x)}{x^{\tau+1 / 2}} d x, \quad \operatorname{Re} \tau>\frac{1}{2}
\end{aligned}
$$

Similarly, the second integral may be written in the form

$$
\begin{aligned}
\int_{1}^{\infty} \frac{a(x)}{x^{\tau+1}} d x & =\sum_{k=0}^{\infty} \int_{4^{k}}^{4^{k+1}} \frac{a(x)}{x^{\tau+1}} d x \\
& =\sum_{k=0}^{\infty} \int_{1}^{4} \frac{a\left(4^{k} x\right)}{2^{2 k \tau} x^{\tau+1}} d x \\
& =\int_{1}^{4} \frac{1}{x^{\tau+1}} \sum_{k=0}^{\infty} \frac{a\left(4^{k} x\right)}{2^{2 k \tau}} d x
\end{aligned}
$$

To evaluate the integrand, we need the following result.

LEMMA 6. In the notation of (2.5) and (2.6) we have that (6.5) $a\left(4^{k} x\right)=2^{k} a(x)-\sum_{r=1}^{k} \rho\left(d_{r}\right) a\left(b_{r}\right) 2^{k-r}$, for $x>0, k \geq 1$.

Proof. From (2.14) we have $\lambda\left(4^{k} x\right)=\lambda(x)$, so from (2.13) we find that

$$
s\left(4^{k} x\right)-2^{k} s(x)=a\left(4^{k} x\right)-2^{k} a(x) .
$$

Equation (6.5) is now immediate from (2.9).

With (6.5) we can write the infinite sum in (6.4) as follows:

$$
\begin{aligned}
\sum_{k=0}^{\infty} \frac{a\left(4^{k} x\right)}{2^{2 k \tau}} & =\sum_{k=0}^{\infty} \frac{a(x)}{2^{k(2 \tau-1)}}-\sum_{k=1}^{\infty} 2^{-2 k \tau} \sum_{r=1}^{\infty} \rho\left(d_{r}\right) a\left(b_{r}\right) 2^{k-r} \\
& =\left(1-2^{1-2 \tau}\right)^{-1} a(x)-\sum_{r=1}^{\infty} \rho\left(d_{r}\right) a\left(b_{r}\right) 2^{-r} \sum_{k=r}^{\infty} 2^{k(1-2 \tau)} \\
& =\left(1-2^{1-2 \tau}\right)^{-1} a(x)-\left(1-2^{1-2 \tau}\right)^{-1} a_{2 \tau}(x),
\end{aligned}
$$

where $a_{\tau}(x)$ is defined by (3.2). 
Putting the results of (6.6), (6.4), and (6.3) into (6.2) gives finally that

$$
\begin{aligned}
& \left(1-2^{1-2 \tau}\right) \eta(\tau) \\
& \quad=2^{1-2 \tau}-1+\tau \int_{1}^{4} \frac{\lambda(x)}{x^{\tau+1 / 2}} d x+\tau \int_{1}^{4} \frac{a(x)-a_{2 \tau}(x)}{x^{\tau+1}} d x
\end{aligned}
$$

initially for $\operatorname{Re} \tau>1 / 2$. But the integrals in this formula define analytic functions of $\tau$ for $\operatorname{Re} \tau>0$. (In fact the first integral is entire.) Thus (6.7) defines the analytic continuation of $\eta(\tau)$ to the half-plane $\operatorname{Re} \tau>0$, and $\eta$ has at most simple poles at the points $\tau$ for which $2^{1-2 \tau}=1$, i.e. the points $\gamma_{n}=1 / 2+\pi n i / \log 2, n \in \mathbf{Z}$. This proves

THEOREM 10. The function $\eta(\tau)^{\circ}$ defined by (6.1) has a meromorphic continuation to the half-plane $\operatorname{Re} \tau>0$, with at most simple poles at the points $\gamma_{n}=1 / 2+\pi n i / \log 2, n \in \mathbf{Z}$.

In fact, the function $\eta(\tau)$ has a meromorphic continuation to the whole complex plane, but we shall not give the proof of this fact here. Rather, we point out the following connection between $c_{n}$ and the behavior of $\eta(\tau)$ at the point $\tau=\gamma_{n}$.

THEOREM 11. The $n$th Fourier coefficient $c_{n}$ of $\lambda(x)$ is related to the residue $R_{n}$ of $\eta(\tau)$ at $\gamma_{n}$ by the formula

$$
c_{n}=R_{n} / \gamma_{n}=\eta_{0}\left(\gamma_{n}\right) /\left(\gamma_{n} \log 4\right)
$$

where $\eta_{0}(\tau)=\left(1-2^{1-2 \tau}\right) \eta(\tau)$

Proof. Since $2^{2 \gamma_{n}}=2$, we have $a_{2 \gamma_{n}}(x)=a(x)$ for all $n \in \mathbf{Z}$ and $x>0$. Putting $\tau=\gamma_{n}$ in (6.7) gives therefore that

$$
\eta_{0}\left(\gamma_{n}\right)=\gamma_{n} \int_{1}^{4} \frac{\lambda(x)}{x^{1 / 2+\gamma_{n}}} d x=\gamma_{n} \log 4 \cdot c_{n} .
$$

Equation (6.8) is immediate from this and the fact that $\eta_{0}\left(\gamma_{n}\right)=\log 4 \cdot R_{n}$.

COROLlaRY 1. Infinitely many of the points $\gamma_{n}$ are simple poles of $\eta(\tau)$. In fact, $R_{n} \neq O\left(|n|^{-1-\delta}\right)$, as $n \rightarrow \pm \infty$, for any $\delta>0$. 
Proof. Immediate from (6.8) and Theorem 9.

Equation (6.8) can also be used to estimate the size of $c_{n}$. To do this we note the Dirichlet series expansion for $\eta_{0}(\tau)$ :

$$
\begin{aligned}
\eta_{0}(\tau) & =\left(1-2^{1-2 \tau}\right) \eta(\tau)=\sum_{n=1}^{\infty} \frac{a(n)}{n^{\tau}}-2 \sum_{n=1}^{\infty} \frac{a(n)}{(4 n)^{\tau}} \\
& =\sum_{n=1}^{\infty} \frac{a^{*}(n)}{n^{\tau}},
\end{aligned}
$$

where

$$
a^{*}(n)= \begin{cases}a(n), & \text { if } 4 \nmid n, \\ -a(n), & \text { if } 4 \mid n\end{cases}
$$

If we set

$$
s^{*}(x)=\sum_{k=0}^{[x]} a^{*}(k),
$$

in analogy to (1.1), then it is easy to see that

$$
\begin{aligned}
s^{*}(x) & =s(x)-2 s(x / 4)=a(x)-2 a(x / 4) \\
& =O(1), \quad \text { as } x \rightarrow \infty .
\end{aligned}
$$

Hence (6.9) converges for $\operatorname{Re} \tau>0$. This implies the following corollary to Theorem 11.

Corollary 2. For any $\delta>0$ we have $c_{n}=O\left(|n|^{-1 / 2+\delta}\right)$.

Proof. We use Satz 33 of Landau [5], p. 784 (with $\alpha=0, \tau=1$, $\delta<1 / 2, \sigma=1 / 2)$ to deduce that

$$
\eta_{0}\left(\gamma_{n}\right)=O\left(|n|^{1 / 2+\delta}\right), \quad \text { for all } \delta>0 .
$$

The corollary is then clear from (6.8).

We conclude this section with a short table of the coefficients $c_{n}$.

\section{TABLE 1}

\begin{tabular}{cccc}
$n$ & $\operatorname{Re} c_{n}$ & $\operatorname{Im} c_{n}$ & $\left|c_{n}\right|$ \\
\hline 0 & 1.5053 & 0 & 1.5053 \\
1 & -.0663 & .0911 & .1126 \\
2 & -.0927 & -.1331 & .1622 \\
3 & .0018 & -.0031 & .0035 \\
4 & .0352 & .0116 & .0370 \\
\hline
\end{tabular}


The values were computed using the first 1,500,000 terms of (6.9) and the formula

$$
\gamma_{n} \cdot \log 4 \cdot c_{n}=\sum_{k=1}^{N} \frac{a^{*}(k)}{k^{\gamma_{n}}}-\frac{s^{*}(N)}{(N+1)^{\gamma_{n}}}+\gamma_{n} \int_{N+1}^{\infty} \frac{s^{*}(x)}{x^{\gamma_{n}+1}} d x,
$$

where $N=1.5 \times 10^{6}$. The total error, due to roundoff and to the integral in this formula, is at most .002 in absolute value, and so $c_{n} \neq 0$ for $0 \leq n \leq 4$.

7. The cumulative distribution. In this section we use the function $\lambda(x)$ to show that the sequence $\{s(n) / \sqrt{n}\}$ has no cumulative distribution function on the interval $(\sqrt{3 / 5}, \sqrt{6})$. Recall the following general definition.

DEFINITION. Let $\left\{u_{n}\right\}$ be a sequence of real numbers contained in an interval $J$, and let $\alpha \in J$. If $D(x, \alpha)$ denotes the number of $n \leq x$ for which $u_{n} \leq \alpha$, and if the $\operatorname{limit}_{\lim _{x \rightarrow \infty}} x^{-1} D(x, \alpha)=D(\alpha)$ exists, then the sequence $\left\{u_{n}\right\}$ is said to have the distribution $D(\alpha)$ at $\alpha . D(\alpha)$ is called the cumulative distribution function of $\left\{u_{n}\right\}$.

THEOREM 12. The cumulative distribution function of $\{s(n) / \sqrt{n}\}$ does not exist at any point of $(\sqrt{3 / 5}, \sqrt{6})$.

Proof. Let $\alpha \in(\sqrt{3 / 5}, \sqrt{6})$, and assume $D(\alpha)$ exists for the sequence $u_{n}=s(n) / \sqrt{n}$ in the above definition.

(a) We first show that $D(\alpha)$ must equal one. By Corollary 1 to Theorem 5 we may choose an $x_{1} \in[1,4]$ for which $\lambda\left(x_{1}\right)<\alpha$. Let $\varepsilon$ be such that $0<\varepsilon<1$ and

$$
\lambda(x)<\alpha \quad \text { when }\left|x-x_{1}\right| \leq \varepsilon,
$$

and set $M=\max _{\left|x-x_{1}\right| \leq \varepsilon} \lambda(x)$. Then $M<\alpha$. Set $\delta=\alpha-M$, and choose $k_{0}$ so large that $2^{-k_{0}}\left(x_{1}-\varepsilon\right)^{-1 / 2}<\delta$. From (2.15) we have for any $x$ satisfying $\left|x-x_{1}\right| \leq \varepsilon$ and for any $k \geq k_{0}$ that $\left|\lambda(x)-s\left(4^{k} x\right) / \sqrt{4^{k} x}\right| \leq$ $2^{-k} x^{-1 / 2} \leq 2^{-k_{0}}\left(x_{1}-\varepsilon\right)^{-1 / 2}<\delta$, so

$$
\frac{s\left(4^{k} x\right)}{\sqrt{4^{k} x}}<\lambda(x)+\delta \leq M+(\alpha-M)=\alpha .
$$

It follows that $s(r) / \sqrt{r}<\alpha$ for every integer $r$ of the interval $4^{k}\left(x_{1}-\varepsilon\right)$ $<r \leq 4^{k}\left(x_{1}+\varepsilon\right)$. But the number of integers in this interval is

$$
4^{k}\left(x_{1}+\varepsilon\right)-4^{k}\left(x_{1}-\varepsilon\right)+O(1)=2 \varepsilon 4^{k}+O(1) .
$$


Thus if we put $x_{k}^{-}=4^{k}\left(x_{1}-\varepsilon\right)$ and $x_{k}^{+}=4^{k}\left(x_{1}+\varepsilon\right)$, we have

$$
D\left(x_{k}^{+}, \alpha\right)=D\left(x_{k}^{-}, \alpha\right)+2 \varepsilon 4^{k}+O(1) \text {. }
$$

Dividing both sides by $x_{k}^{+}=\left(x_{1}+\varepsilon\right) x_{k}^{-} /\left(x_{1}-\varepsilon\right)$ and letting $k \rightarrow \infty$ then gives that

$$
D(\alpha)=D(\alpha) \frac{x_{1}-\varepsilon}{x_{1}+\varepsilon}+\frac{2 \varepsilon}{x_{1}+\varepsilon},
$$

which implies $D(\alpha)=1$, as claimed.

(b) We now show that $D(\alpha)=0$; this will contradict (a) and prove the theorem. We choose an $x_{1} \in[1,4]$ with $\lambda\left(x_{1}\right)>\alpha$, and an $\varepsilon$ for which

$$
0<\varepsilon<1 \text { and } \lambda(x)>\alpha \text { when }\left|x-x_{1}\right| \leq \varepsilon .
$$

We also pick $k_{0}$ so that $2^{-k_{0}}\left(x_{1}-\varepsilon\right)^{-1 / 2}<\delta$, where this time $\delta=m-\alpha$ and $m=\min _{\left|x-x_{1}\right| \leq \varepsilon} \lambda(x)$. As before, we have for any $x$ with $\left|x-x_{1}\right| \leq \varepsilon$ and any $k \geq k_{0}$ that

$$
\left|\lambda(x)-\frac{s\left(4^{k} x\right)}{\sqrt{4^{k} x}}\right|<\delta,
$$

whence

$$
\frac{s\left(4^{k} x\right)}{\sqrt{4^{k} x}}>\lambda(x)-\delta \geq m-(m-\alpha)=\alpha .
$$

Thus $s(r) / \sqrt{r}>\alpha$ for all the integers $r$ in the interval

$$
4^{k}\left(x_{1}-\varepsilon\right)<r \leq 4^{k}\left(x_{1}+\varepsilon\right),
$$

and

$$
D\left(x_{k}^{+}, \alpha\right)=D\left(x_{k}^{-}, \alpha\right),
$$

where $x_{k}^{+}=4^{k}\left(x_{1}+\varepsilon\right)$ and $x_{k}^{-}=4^{k}\left(x_{1}-\varepsilon\right)$. Therefore

$$
\frac{1}{x_{k}^{+}} D\left(x_{k}^{+}, \alpha\right)=\frac{1}{x_{k}^{-}} D\left(x_{k}^{-}, \alpha\right) \cdot \frac{x_{1}-\varepsilon}{x_{1}+\varepsilon},
$$

and letting $k \rightarrow \infty$ shows that

$$
D(\alpha)=D(\alpha) \cdot \frac{x_{1}-\varepsilon}{x_{1}+\varepsilon},
$$

i.e. that $D(\alpha)=0$. 
For the sequence $u_{n}=t(n) / \sqrt{n}$ we have the analogous

THEOREM 13. The cumulative distribution function of the sequence $\{t(n) / \sqrt{n}\}$ does not exist at any point $\alpha \in(0, \sqrt{3})$. However it does exist when $\alpha=0$, and $D(0)=0$.

Proof. The proof that $D(\alpha)$ does not exist for $\alpha$ in $(0, \sqrt{3})$ follows, mutatis mutandis, the proof of Theorem 6. Thus assume that $\alpha=0$. To show $D(0)=0$ we proceed as follows. Let $n_{\nu}$ be the $\nu$ th integer for which $t(n)=0$. Clearly

$$
\frac{1}{n} D(n, 0) \leq \frac{1}{n_{\nu}} D\left(n_{\nu}, 0\right)=\frac{\nu}{n_{\nu}}
$$

if $n_{\nu} \leq n<n_{\nu+1}$, and so it suffices to show that

$$
\nu / n_{\nu} \rightarrow 0 \text { as } \nu \rightarrow \infty \text {. }
$$

However, by the Vorbemerkung in Satz 13 of [1], if

$$
\nu=\sum_{r=0}^{k} \varepsilon_{r} 2^{r}
$$

is the binary representation of $\nu$, then

$$
n_{\nu}=\sum_{r=0}^{k} \varepsilon_{r} 2^{2 r+1}-1 .
$$

Thus $n_{\nu} \geq 2^{2 k+1}-1>\frac{1}{2} \nu^{2}-1$, and so $\nu / n_{\nu} \leq 2 \nu /\left(\nu^{2}-2\right) \rightarrow 0$ as $\nu \rightarrow \infty$.

As the above proofs show, the nonexistence of the cumulative distribution functions is attributable to the fact that the sequences $s(n) / \sqrt{n}$ and $t(n) / \sqrt{n}$ behave very "sluggishly".

8. The logarithmic distribution. It is possible to show that a modified distribution function does exist for the sequences $\{s(n) / \sqrt{n}\}$ and $\{t(n) / \sqrt{n}\}$. The type of distribution we consider is defined as follows.

DEFINITION. Let $\left\{u_{n}\right\}$ be a real sequence contained in an interval $J$, and let $\alpha \in J$. If

$$
L(x, \alpha)=\sum_{\substack{1 \leq n \leq x \\ u_{n} \leq \alpha}} \frac{1}{n},
$$


and if the limit

$$
\lim _{x \rightarrow \infty} \frac{1}{\log x} L(x, \alpha)=L(\alpha)
$$

exists, then the sequence $\left\{u_{n}\right\}$ is said to have the logarithmic distribution $L(\alpha)$ at $\alpha . L(\alpha)$ is called the logarithmic distribution function of the sequence.

We shall prove that both sequences $\{s(n) / \sqrt{n}\}$ and $\{t(n) / \sqrt{n}\}$ have logarithmic distribution functions which are defined everywhere in the respective intervals $[\sqrt{3 / 5}, \sqrt{6}]$ and $[0, \sqrt{3}]$. We need a lemma.

LEMma 7. Let $\alpha \in[\sqrt{3 / 5}, \sqrt{6}]$ be fixed and let $S_{\alpha}$ denote the set $S_{\alpha}=\{x: 1 \leq x \leq 4$ and $\lambda(x)=\alpha\}$. Then $S_{\alpha}$ has measure zero.

Proof. Let $x_{0}=\sum_{r=0}^{\infty} d_{r} 4^{-r}$ be an element of $S_{\alpha}$ which is normal to the base 4. Choose an $n \geq 1$ for which $d_{j}=0$ for $n \leq j \leq n+3$, and set

$$
x_{n}=x_{0}+4^{-n}, \quad y_{n}=x_{0}+4^{-n}+4^{-n-3}=x_{0}+h_{n} .
$$

As in the proof of Theorem 6 we have that $\left|a\left(x_{0}\right)-a\left(x_{n}\right)\right|=2^{-n}$. Now if $x^{*}$ satisfies $x_{n}<x^{*}<y_{n}$, it is easy to see that $x^{*}=\sum_{r=0}^{\infty} d_{r}^{*} 4^{-r}$, with

$$
d_{r}^{*}= \begin{cases}d_{r}, & r<n \\ 1, & r=n, \\ 0, & r=n+1, n+2\end{cases}
$$

Thus

$$
\begin{aligned}
\left|a\left(x^{*}\right)-a\left(x_{n}\right)\right| & =\left|\sum_{r=n+3}^{\infty} \frac{\rho\left(d_{r}\right) a\left(b_{r}\right)-\rho\left(d_{r}^{*}\right) a\left(b_{r}^{*}\right)}{2^{r}}\right| \\
& \leq \sum_{r=n+3}^{\infty} 2^{1-r}=2^{-n-1},
\end{aligned}
$$

and it follows that

$$
\begin{aligned}
\left|a\left(x_{0}\right)-a\left(x^{*}\right)\right| & =\left|a\left(x_{0}\right)-a\left(x_{n}\right)+a\left(x_{n}\right)-a\left(x^{*}\right)\right| \\
& \geq 2^{-n}-2^{-n-1}=2^{-n-1} .
\end{aligned}
$$

Furthermore, equation (5.7) implies

$$
\begin{aligned}
\left|\lambda\left(x_{0}\right)-\lambda\left(x^{*}\right)\right| & =\left|\frac{a\left(x^{*}\right)-a\left(x_{0}\right)}{\sqrt{x^{*}}}+O\left(\left|x^{*}-x_{0}\right|\right)\right| \\
& \geq \kappa_{0} 2^{-n}-\kappa_{1} 4^{-n} \geq \kappa_{2} 2^{-n}, \quad \text { for } n \geq n_{0},
\end{aligned}
$$


where $\kappa_{0}, \kappa_{1}, \kappa_{2}$ are positive constants and $n_{0}$ is sufficiently large. Therefore, for $n \geq n_{0}$ satisfying $d_{n}=d_{n+1}=d_{n+2}=d_{n+3}=0$, we have

$$
\lambda\left(x^{*}\right) \neq \alpha \quad \text { for } x_{n}<x^{*}<x_{n}+4^{-n-3} .
$$

If $m$ denotes Lebesgue measure, we deduce

$$
\frac{1}{h_{n}} m\left(S_{\alpha} \cap\left(x_{0}, x_{0}+h_{n}\right)\right) \leq \frac{4^{-n}}{h_{n}}=\frac{64}{65}<1,
$$

for an infinite sequence of $h_{n}$ 's tending to zero.

On the other hand, if $\chi_{\alpha}$ denotes the characteristic function of the set $S_{\alpha}$, then

$$
\begin{aligned}
& \frac{1}{h} m\left(S_{\alpha} \cap\left(x_{0}, x_{0}+h\right)\right) \\
& \quad=\frac{1}{h} \int_{x_{0}}^{x_{0}+h} \chi_{\alpha}(t) d t \rightarrow \chi_{\alpha}\left(x_{0}\right), \quad \text { as } h \rightarrow 0,
\end{aligned}
$$

for almost all $x_{0}$ (see [4], p. 173). Equation (8.1) shows therefore that all normal numbers $x_{0}$ in $S_{\alpha}$ lie in the null set of exceptional numbers for which (8.2) does not hold, since for these $x_{0}, \chi_{\alpha}\left(x_{0}\right)=1$. But this implies $m\left(S_{\alpha}\right)=0$.

The argument in the above lemma is due to A. J. E. M. Janssen (private communication).

We can now prove

THEOREM 14. If $\alpha \in[\sqrt{3 / 5}, \sqrt{6}]$, then the logarithmic distribution function of the sequence $\{s(n) / \sqrt{n}\}$ exists at $\alpha$, and has the value

$$
L(\alpha)=\frac{1}{\log 4} \int_{E_{\alpha}} \frac{1}{x} d x
$$

where $E_{\alpha}$ is the set

$$
E_{\alpha}=\{x: 1 \leq x \leq 4 \text { and } \lambda(x) \leq \alpha\} .
$$

Proof. Let $I_{k}$ denote the set of integers $r$ contained in the interval $4^{k} \leq r<4^{k+1}, k \geq 0$, and consider the sum

$$
\sigma_{k}(\alpha)=\sum_{\substack{r \in I_{k} \\ \lambda(r) \leq \alpha}} \frac{1}{r}=\sum_{r=4^{k}}^{4^{k+1}-1} \frac{\omega_{\alpha}\left(r / 4^{k}\right)}{r},
$$


where $\omega_{\alpha}$ is the characteristic function of the set $E_{\alpha}$. Note that $\sigma_{k}(\alpha)$ is just a Riemann sum for the function $\omega_{\alpha}(x) / x$ on the interval [1,4], since

$$
\sigma_{k}(\alpha)=\sum_{r=4^{k}}^{4^{k+1}-1} \frac{\omega_{\alpha}\left(r / 4^{k}\right)}{r / 4^{k}} 4^{-k} .
$$

Now $\lambda$ is a continuous function, so it is clear from (8.4) that the discontinuities of $\omega_{\alpha}$ are contained in the set $S_{\alpha}=\{x: 1 \leq x \leq 4$ and $\lambda(x)=\alpha\}$. By Lemma 7, $S_{\alpha}$ has measure zero, and therefore $\omega_{\alpha}$ is Riemann integrable. (See [4], p. 64.) Consequently,

$$
h(\alpha)=\lim _{k \rightarrow \infty} \sigma_{k}(\alpha)=\int_{1}^{4} \frac{\omega_{\alpha}(x)}{x} d x=\int_{E_{\alpha}} \frac{1}{x} d x .
$$

Note also that $h(\alpha)$ is a continuous function of $\alpha$, since the set $E_{\alpha+\varepsilon}$ tends to the set $E_{\alpha}$ as $\varepsilon \rightarrow 0^{+}$, and since $E_{\alpha-\varepsilon}$ tends to $E_{\alpha}-S_{\alpha}$ as $\varepsilon \rightarrow 0^{+}$, which differs from $E_{\alpha}$ by the null set $S_{\alpha}$.

This fact implies easily that

$$
\lim _{k \rightarrow \infty} \sigma_{k}\left(\alpha-2^{-k}\right)=\lim _{k \rightarrow \infty} \sigma_{k}\left(\alpha+2^{-k}\right)=h(\alpha) .
$$

For instance, if $k_{0}$ is fixed and $k \geq k_{0}$, we have

$$
\sigma_{k}\left(\alpha-2^{-k_{0}}\right) \leq \sigma_{k}\left(\alpha-2^{-k}\right) \leq \sigma_{k}\left(\alpha+2^{-k}\right) \leq \sigma_{k}\left(\alpha+2^{-k_{0}}\right) .
$$

Thus by (8.5),

$$
\begin{aligned}
h\left(\alpha-2^{-k_{0}}\right) & \leq \liminf _{k \rightarrow \infty} \sigma_{k}\left(\alpha-2^{-k}\right) \leq \limsup _{k \rightarrow \infty} \sigma_{k}\left(\alpha+2^{-k}\right) \\
& \leq h\left(\alpha+2^{-k_{0}}\right) .
\end{aligned}
$$

But for large $k_{0}$, both sides of this inequality can be made arbitrarily close to $h(\alpha)$, and this proves (8.6).

We now show that the limit of

$$
\overline{\boldsymbol{\sigma}}_{k}(\alpha)=\sum_{\substack{r \in I_{k} \\ s(r) \leq \alpha \sqrt{r}}} \frac{1}{r},
$$

as $k \rightarrow \infty$, is $h(\boldsymbol{\alpha})$. From (2.15) we have

$$
\left|\lambda(r)-\frac{s(r)}{\sqrt{r}}\right| \leq \frac{1}{\sqrt{r}} \leq 2^{-k}, \quad \text { for } r \in I_{k},
$$


and so

$$
\begin{array}{ll}
\frac{s(r)}{\sqrt{r}} \leq \alpha & \text { implies } \lambda(r) \leq \alpha+2^{-k}, \\
\lambda(r) \leq \alpha-2^{-k} & \text { implies } \frac{s(r)}{\sqrt{r}} \leq \alpha,
\end{array}
$$

for these $r$. It follows that

$$
\sigma_{k}\left(\alpha-2^{-k}\right) \leq \bar{\sigma}_{k}(\alpha) \leq \sigma_{k}\left(\alpha+2^{-k}\right) .
$$

Letting $k \rightarrow \infty$ and using (8.6) then gives

$$
\lim _{k \rightarrow \infty} \bar{\sigma}_{k}(\alpha)=h(\alpha) \text {. }
$$

Thus we have also

$$
\lim _{m \rightarrow \infty} \frac{1}{m} \sum_{k=0}^{m-1} \bar{\sigma}_{k}(\alpha)=h(\alpha)
$$

since the $(C, 1)$ method is regular.

Finally, suppose that $n \geq 1$ is arbitrary and $m$ is chosen so that $4^{m} \leq n<4^{m+1}$. Then $m=[\log n / \log 4]$, and

$$
\frac{m}{\log n} \cdot \frac{1}{m} \sum_{k=0}^{m-1} \bar{\sigma}_{k}(\alpha) \leq \frac{1}{\log n} \sum_{\substack{r=1 \\ s(r) \leq \alpha \sqrt{r}}}^{n} \frac{1}{r} \leq \frac{m+1}{\log n} \cdot \frac{1}{m+1} \sum_{k=0}^{m} \bar{\sigma}_{k}(\alpha) .
$$

Hence by (8.7),

$$
\lim _{n \rightarrow \infty} \frac{1}{\log n} \sum_{\substack{r=1 \\ s(r) \leq \alpha \sqrt{r}}}^{n} \frac{1}{r}=\frac{1}{\log 4} h(\alpha)=\frac{1}{\log 4} \int_{E_{\alpha}} \frac{1}{x} d x
$$

and this proves (8.3).

THEOREM 15. If $\alpha \in[0, \sqrt{3}]$, then the logarithmic distribution function of the sequence $\{t(n) / \sqrt{n}\}$ exists at $\alpha$, and has the value

$$
L^{*}(\alpha)=\frac{1}{\log 4} \int_{E_{\alpha}^{*}} \frac{1}{x} d x
$$

where $E_{\alpha}^{*}=\{x: 1 \leq x \leq 4$ and $\mu(x) \leq \alpha\}$.

Proof. The theorem is proved by exactly the same argument used to prove Theorem 14, the crucial point being that the set $S_{\alpha}^{*}=\{x: 1 \leq x \leq 4$ and $\mu(x)=\alpha\}$ has measure zero. We omit the details. 


\section{REFERENCES}

[1] J. Brillhart and P. Morton, Über Summen von Rudin-Shapiroschen Koeffizienten, Illinois J. Math., 22 (1978), 126-148.

[2] L. Carleson, On convergence and growth of partial sums of Fourier series, Acta. Math., 116 (1966), 135-157.

[3] G. H. Hardy and W. W. Rogosinski, Fourier Series, Cambridge Tracts in Mathematics and Mathematical Physics, No. 38, 1968.

[4] H. Kestelman, Modern Theories of Integration, Dover Publications, New York, 1960.

[5] E. Landau, Handbuch der Lehre von der Verteilung der Primzahlen, Chelsea Publishing Co., 1974.

[6] I. Niven, Irrational Numbers, Carus Mathematical Monographs, No. 11, 1956.

Received January 13, 1981.

UNIVERSITY OF ARIZONA

TusCON, AZ 85721

The Hungarian ACademy of Sciences

BUDAPEST, HUNGARY

California Institute of TeChNology

PASADENA, CA 91125

AND

HARVARD UNIVERSITY

CAMBRIDGE, MA 02138 
